ИЗВЕСТИЯ АКАДЕМИИ НАУК ЭСТОНСКОИ ССР. ФИЗИКА * МАТЕМАТИКА

PROCEEDINGS OF THE ACADEMY OF SCIENCES OF THE ESTONIAN SSR.

PHYSICS * MATHEMATICS

$1987,36,3$

УДК $517.988: 519.615$

O. ВАAPMAHH

\title{
О МЕТОДАХ ТИПА КАСАТЕЛЬНЫХ ГИПЕРБОЛ
}

\author{
(Представил Н. Алумяэ)
}

Пусть дано уравнение

$$
F(x)=0,
$$

где дважды дифференцируемый оператор $F(x)$ действует из одного банахова пространства $X$ в другое $Y$. Для решения этого уравнения можно применить метод касательных гипербол

$$
x_{k+1}=x_{k}-Q_{k}^{-1} \Gamma_{k} F\left(x_{k}\right), \quad k=0,1, \ldots,
$$

где

$$
\Gamma_{k}=\left[F^{\prime}\left(x_{k}\right)\right]^{-1} \quad \text { и } \quad Q_{k}=I-\frac{1}{2} \Gamma_{k} F^{\prime \prime}\left(x_{k}\right) \Gamma_{k} F\left(x_{k}\right)
$$

Однако во многих случаях применение основного метода (2) нецелесообразно из-за трудоемкости вычисления второй производной $F^{\prime \prime}(x)$. Поэтому представляют интерес модификации метода (2), в которых вместо оператора $F^{\prime \prime}(x)$ используется его некоторая вычисляемая просто аппроксимация.

Целью настоящей работы является исследование общих схем итерационных методов третьего порядка, включающих как уже известные, так и новые алгоритмы типа (2). Изучаются варианты метода (2), в которых вместо операторов $F^{\prime \prime}(x), Q^{-1}(x)$ и $\Gamma(x)$ используются некоторые их приближения. В частности, эти вопросы изучены в работах $\left[{ }^{1-7}\right]$.

Если выражение $F^{\prime \prime}(x)(y-x)$ заменить на $L(x, y-x)$, которая аппроксимирует $F^{\prime \prime}(x)(y-x)$ в рассматриваемой области $S, x, y \in S$, и вместо $\Gamma(x)$ использовать также некоторое его приближение, то метод (2) превращается в метод

$$
x_{k+1}=x_{k}-\left[F^{\prime}\left(x_{k}\right)+\frac{1}{2} L\left(x_{k}, y_{k}-x_{k}\right)\right]^{-1} F\left(x_{k}\right)
$$

или

$$
x_{k+1}=x_{k}-U_{k}^{-1} A_{k} F\left(x_{k}\right) \text {, }
$$

где $U_{k}=A_{k} F^{\prime}\left(x_{k}\right)+\frac{1}{2} A_{k} L\left(x_{k}, y-x_{k}\right), y_{k}-x_{k}=-A_{k} F\left(x_{k}\right)$.

Если вместо $U_{k}{ }^{-1}$, в свою очередь, принять приближенный к нему оператор $V_{k}$, то получается

$$
x_{k+1}=x_{k}-V_{k} A_{k} F\left(x_{k}\right), \quad k=0,1, \ldots .
$$

1. В дальнейшем предполагается существование и ограниченность операторов $\left[F^{\prime}(x)\right]^{-1}$ и $U^{-1}(x)$. Если при этом $\gamma_{k}<1$ и $\bar{\gamma}_{k}<1$ (см. 
ниже), то по теореме Банаха существуют также ограннченные операторы $A_{k}^{-1}$ и $V_{k}^{-1}$.

Введем последовательности чисел $\left\{\gamma_{k}\right\},\left\{\bar{\gamma}_{k}\right\},\left\{\eta_{k}\right\}$ и постоянные $\lambda, \Lambda, M, M_{1}$ и $H$, удовлетворяющие неравенствам

$$
\begin{gathered}
\left\|I-A_{k} F^{\prime}\left(x_{k}\right)\right\| \leqslant \gamma_{k}, \quad\left\|I-V_{k} U_{k}\right\| \leqslant \bar{\gamma}_{k}, \quad\left\|V_{k} A_{k} F\left(x_{k}\right)\right\| \leqslant \eta_{k}, \\
\left\|F^{\prime}(x)\right\| \leqslant M, \quad\left\|F^{\prime \prime}(x)\right\| \leqslant K, \quad\left\|A_{k}\right\| \leqslant \lambda, \quad\left\|V_{k}\right\| \leqslant \Lambda \\
\left\|V_{k}^{-1}\right\| \leqslant M_{1}, \quad\left\|A_{k+1} A_{k}^{-1}\right\| \leqslant H, \quad k=0,1, \ldots .
\end{gathered}
$$

Т е о р ем а 1. Пусть $x_{0} \in X, S=\left\{x \in X:\left\|x-x_{0}\right\| \leqslant \mathrm{e}\right\}$ и на $S$ выполнены следующие условия:

$1^{\circ}$ оператор $F(x)$ дважды дифференцируем (по Фреше);

$2^{\circ}$ вторая производная $F^{\prime \prime}(x)$ удовлетворяет условию Липшица

$$
\left\|F^{\prime \prime}(x)-F^{\prime \prime}(y)\right\| \leqslant L_{2}\|x-y\| \quad(x, y \in S) ;
$$

$3^{\circ}\left\|F^{\prime \prime}(x)(y-x)-L(x, y-x)\right\| \leqslant G\|x-y\|^{2}, \quad\|L(x, y-x)\| \leqslant$

$$
\leqslant G_{1}\|x-y\|, \quad\left(G, G_{1}<\infty\right) ;
$$

$4^{\circ}$ существуют $\Gamma(x), U^{-1}(x) u\|\Gamma(x)\| \leqslant C,\left\|U^{-1}(x)\right\| \leqslant C_{1}, \quad\left(C, C_{1}<\infty\right)$;

$5^{\circ} \delta=\delta_{0}<1$ (ниже величина $\delta_{0}$ определяется по разному в случаях 1) -3$))$.

Тогда:

1) Если $\gamma_{k} \leqslant \gamma_{0}<1, \overline{\gamma_{k}} \leqslant \gamma_{0}<1$ u $r_{1}=\eta_{0} /\left(1-\delta_{0}\right) \leqslant \mathrm{Q}, \quad$ то уравнение (1) имеет в $S$ решение $x^{*}, \kappa$ которому сходится последовательность (5), причем $\left\|x^{*}-x_{0}\right\| \leqslant r_{1} u$

$$
\left\|x_{k}-x^{*}\right\| \leqslant r_{1} \delta^{k}
$$

где $\quad \delta_{0}=\Lambda\left[H M_{1} \bar{\gamma}_{0}+\frac{1}{2} \lambda K\left(\gamma_{0}+M_{1} \bar{\gamma}_{0}\right) \eta_{0}+\frac{1}{2} \lambda\left(G M_{1}+\frac{1}{2} \lambda K G_{1} M_{1}+\right.\right.$ $\left.\left.+\frac{1}{3} L^{2}\right) \eta_{0}^{2}\right]$. Если $\bar{\gamma}_{0} \geqslant \bar{\gamma}_{1} \geqslant \ldots \geqslant \bar{\gamma}_{k} \geqslant \ldots \geqslant 0 u \bar{\gamma}_{k} \rightarrow 0$ nрu $\mathrm{k} \rightarrow \infty$, то со сверхлинейной скоростью

$$
\left\|x_{k}-x^{*}\right\| \leqslant r_{1} \prod_{i=0}^{k-1} \delta_{i}
$$

где $\delta_{i}=\Lambda\left[H M_{1} \bar{\gamma}_{i}+\frac{1}{2} \lambda K\left(\gamma_{i}+M_{1} \bar{\gamma}_{i}\right) \eta_{i}+\frac{1}{2} \lambda\left(G M_{1}^{2}+\frac{1}{2} \lambda K G_{1} M_{1}+\frac{1}{3} L_{2}\right) \eta_{i}^{2}\right]$.

2) Если $\bar{\gamma}_{k}=C_{2}\left\|x_{k+1}-x_{k}\right\|, C_{2}<\infty \quad$ и $\gamma_{k}<1$, но $\gamma_{k}$ не является бесконечно малой величиной порядка $O\left(\left\|x_{k+1}-x_{k}\right\|\right)$ u $r_{2}=H_{0}(\delta) / d \leqslant \varrho$, где $H_{k}(\delta)=\sum_{i=k}^{\infty} \delta^{2^{\prime}}, \delta=\delta_{0}=\eta_{0} d_{0}, d=\lim _{k \rightarrow \infty} d_{k}, d_{k}=\Lambda\left\{\frac{1}{2} \lambda\left[K \gamma_{k}+\left(G M_{1}^{2}+\right.\right.\right.$ $\left.\left.\left.+\frac{1}{2} \lambda K G_{1} M_{1}+\frac{1}{3} L_{2}\right) \eta_{k}\right]+H M_{1} C_{2} \eta_{k}+\frac{1}{2} \lambda K M_{1} C_{2} \eta_{k}^{2}\right\}$, то уравнение имеет в $S$ решение $x^{*}, \kappa$ которому сходится последовательность (5), причем $\left\|x^{*}-x_{0}\right\| \leqslant r_{2} u$

$$
\left\|x_{k}-x^{*}\right\| \leqslant H_{k}(\delta) / d .
$$

3) Ecлu $\bar{\gamma}_{k}=C_{2}\left\|x_{k+1}-x_{k}\right\|^{2}, \quad \gamma_{k}=C_{3}\left\|x_{k+1}-x_{k}\right\| \quad u \quad r_{3}=H_{0}(\delta) / d \leqslant \varrho$, $\delta_{0}=\eta_{0} \sqrt{d_{0}}, \quad$ əде $\quad d_{k}=d+\frac{1}{2} \lambda \Lambda K M_{1} C_{2} \eta_{k}, \quad d=\Lambda\left[H M_{1} C_{2}+\frac{1}{2} \lambda K C_{3}+\right.$ $\left.+\frac{1}{2} \lambda\left(G M_{1}^{2}+\frac{1}{2} \lambda K G_{1} M_{1}+\frac{1}{3} L_{2}\right)\right]$, 
то последовательность (5) сходится с кубичной скоростью сходимости $\kappa$ решению $x^{*}$ уравнения (1), причем $\left\|x^{*}-x_{0}\right\| \leqslant r_{3} u$

$$
\left\|x_{k}-x^{*}\right\| \leqslant H_{k}(\delta) / \sqrt{d}, \quad H_{k}(\delta)=\sum_{i=k}^{\infty} \delta^{3^{t}} .
$$

Д о к а з а тель с т о. По формуле Тейлора

$$
F\left(x_{k+1}\right)=A_{k}^{-1} V_{k}^{-1}\left(V_{k} U_{k}-I\right)\left(x_{k+1}-x_{k}\right)+R_{k},
$$

где

$$
\begin{gathered}
R_{k}=\frac{1}{2}\left\{\left[F^{\prime \prime}\left(x_{k}\right)\left(y_{k}-x_{k}\right)-L\left(x_{k}, y_{k}-x_{k}\right)\right]\left(x_{k+1}-x_{k}\right)+\right. \\
\left.+F^{\prime \prime}\left(x_{k}\right)\left(y_{k}-x_{k+1}\right)\left(x_{k+1}-x_{k}\right)\right\}+\int_{0}^{1}\left[F^{\prime \prime}\left(x_{k}+t\left(x_{k+1}-x_{k}\right)\right)-\right. \\
\left.-F^{\prime \prime}\left(x_{k}\right)\right]\left(x_{k+1}-x_{k}\right)^{2}(1-t) \mathrm{dt} .
\end{gathered}
$$

Далее,

$$
\begin{aligned}
& x_{k+1}-y_{k}=x_{k+1}-x_{k}-V_{k}^{-1}\left(x_{k+1}-x_{k}\right)= \\
& =\left[I-U_{k}+V_{k}^{-1}\left(V_{k} U_{k}-I\right)\right]\left(x_{k+1}-x_{k}\right) .
\end{aligned}
$$

С учетом (4), (9) и (10) имеем

$$
\left\|I-U_{k}\right\| \leqslant \gamma_{k}+\frac{1}{2}\left\|A_{k} L\left(x_{k}, y_{k}-x_{k}\right)\right\| \leqslant \gamma_{k}+\frac{1}{2} \lambda G_{1}\left\|y_{k}-x_{k}\right\| \leqslant
$$

$$
\leqslant \gamma_{k}+\frac{1}{2} \lambda G_{1} M_{1}\left\|x_{k+1}-x_{k}\right\|,
$$

$\left\|x_{k+1}-x_{k}\right\|=\left\|V_{k} A_{k} F\left(x_{k}\right)\right\| \leqslant\left\|V_{k}\right\|\left[\bar{\gamma}_{k-1}\left\|A_{k} A_{k-1}\right\|\left\|V_{k v-1}^{-1}\right\|\left\|x_{k}-x_{k-1}\right\|+\right.$ $+\frac{1}{2}\left\|A_{k-1}\right\|\left(\gamma_{k-1} K+\bar{\gamma}_{k-1} K\left\|V_{k-1}^{-1}\right\|\right)\left\|x_{k}-x_{k-1}\right\|^{2}+\frac{1}{2}\left\|A_{k-1}\right\|\left(\frac{1}{2} \lambda K G_{1}\left\|V_{k-1}^{-1}\right\|+\right.$

$$
\left.\left.+G\left\|V_{k-1}^{-1}\right\|^{2}+\frac{1}{3} L_{2}\right)\left\|x_{k}-x_{k-1}\right\|^{3}\right]
$$

1) Если $\bar{\gamma}_{k} \leqslant \bar{\gamma}_{0}<1$ и $\gamma_{k} \leqslant \gamma_{0}<1$, то $\left\|x_{k+1}-x_{k}\right\| \leqslant \delta_{k-1}\left\|x_{k}-x_{k-1}\right\|$, где

$$
\begin{aligned}
\delta_{k-1} & =\Lambda\left[H M_{1} \bar{\gamma}_{k-1}+\frac{1}{2} \lambda K\left(\gamma_{k-1}+M_{1} \bar{\gamma}_{k-1}\right) \eta_{k-1}+\right. \\
+ & \left.\frac{1}{2} \lambda\left(G M_{1}^{2}+\frac{1}{2} \lambda K G_{1} M_{1}+\frac{1}{3} L_{2}\right) \eta_{k-1}^{2}\right]
\end{aligned}
$$

и для $n \geqslant k$

$$
\left\|x_{n}-x_{k}\right\| \leqslant \sum_{i=k}^{n-1}\left\|x_{i+1}-x_{i}\right\| \leqslant\left\|x_{1}-x_{0}\right\| \sum_{i=k}^{n-1} \delta^{i}=r_{1}\left(\delta^{k}-\delta^{n}\right) .
$$

Таким образом, последовательность (5) фундаментальна, и справедливы следующие соотношения

$$
\begin{gathered}
x^{*}=\lim _{k \rightarrow \infty} x_{k}, \quad F\left(x^{*}\right)=0, \quad\left\|x_{k}-x^{*}\right\| \leqslant r_{1} \delta^{k}, \quad r_{1}=\eta_{0} /(1-\delta), \\
\left\|x_{k}-x_{0}\right\| \leqslant r_{1}\left(1-\delta^{k}\right) \leqslant r_{1} \leqslant \varrho, \quad \text { т. е. все } x_{k} \in S .
\end{gathered}
$$


Перейдем теперь к дока́зательству случаев 2) и 3). Допустим, чтоิ где

$$
\left\|x_{k+1}-x_{k}\right\| \leqslant d_{k-1}\left\|x_{k}-x_{k-1}\right\|^{p} \quad(p \geqslant 2),
$$

$$
d_{0} \geqslant d_{1} \geqslant \ldots \geqslant d_{k} \geqslant \ldots>0, \quad \lim _{k \rightarrow \infty} d_{k}=d,
$$

тогда имеет место

$$
\begin{gathered}
\left\|x_{k+1}-x_{k}\right\| \leqslant d_{k-1}\left\|x_{k}-x_{k-1}\right\|^{p} \leqslant d^{-\frac{1}{p-1}}\left(d^{\frac{1}{{ }_{k-1}^{p-1}}} \eta_{k-1}\right)^{p} \leqslant \ldots \leqslant d^{-\frac{1}{p-1}} \delta^{p^{k}}, \\
\left\|x_{n}-x_{k}\right\| \leqslant d^{-\frac{1}{p-1}}\left[H_{k}(\delta)-H_{n}(\delta)\right] \quad(n \geqslant k),
\end{gathered}
$$

где $\delta=\eta_{0} \frac{1}{d_{0}^{p-1}}, H_{k}(\delta)=\sum_{i=k}^{\infty} \delta^{p^{t}} ; \quad$ т. е. последовательность $\left\{x_{k}\right\}$ фундаментальна. Следовательно,

$$
\begin{gathered}
x^{*}=\lim _{k \rightarrow \infty} x_{k}, \quad F\left(x^{*}\right)=0, \quad\left\|x_{k}-x^{*}\right\| \leqslant d^{-\frac{1}{p-1}} H_{k}(\delta), \\
\left\|x_{0}-x^{*}\right\| \leqslant d^{-\frac{1}{p-1}} H_{0}(\delta) .
\end{gathered}
$$

В частности, если $\bar{\gamma}_{k-1}=C_{2}\left\|x_{k}-x_{k-1}\right\|^{2}$, а $\gamma_{k-1}$ не является малой величиной порядка $O\left(\left\|x_{k}-x_{k-1}\right\|\right)$ и $\lim _{k \rightarrow \infty} d_{k}=d>0$, то из (11) следует, что $\left\|x_{k+1}-x_{k}\right\| \leqslant d_{k-1}\left\|x_{k}-x_{k-1}\right\|^{2}$,

где

$$
\begin{gathered}
d_{k-1}=\Lambda\left\{\frac{1}{2} \lambda\left[K \gamma_{k-1}+\left(G M_{1}^{2}+\frac{1}{2} \lambda K G_{1} M_{1}+\frac{1}{3} L_{2}\right) \eta_{k-1}\right]+\right. \\
\left.+H M_{1} C_{2} \eta_{k-1}+\frac{1}{2} \lambda K M_{1} C_{2} \eta_{k-1}^{2}\right\}
\end{gathered}
$$

и

$$
H_{k}(\delta)=\sum_{i=k}^{\infty} \delta^{2}
$$

В случае $\bar{\gamma}_{k-1}=C_{2}\left\|x_{k}-x_{k-1}\right\|^{2}, \gamma_{k-1}=C_{3}\left\|x_{k}-x_{k-1}\right\|$ имеем

$$
\begin{array}{cl}
\left\|x_{k+1}-x_{k}\right\| \leqslant d_{k-1}\left\|x_{k}-x_{k-1}\right\|^{3}, & \text { где } \quad d=\Lambda\left[H M_{1} C_{2}+\frac{1}{2} \lambda K C_{3}+\right. \\
\left.+\frac{1}{2} \lambda\left(G M_{1}^{2}+\frac{1}{2} \lambda K G_{1} M_{1}+\frac{1}{3} L_{2}\right)\right], & d_{k-1}=d+\frac{1}{2} \lambda \Lambda K M_{1} C_{2} \eta_{k-1} .
\end{array}
$$

2. Теорема 1 позволяет не только обсудить устойчивости (в смысле сохранения порядка скорости сходимости), а также изучить важные с точки зрения практики виды метода (5).

В $\left.{ }^{4}\right]$ предлагается в качестве $L\left(x_{k}, y_{k}-x_{k}\right)$ брать

$$
2 \beta\left[F^{\prime}\left(x_{k}-\frac{1}{2 \beta} \Gamma_{k} F\left(x_{k}\right)\right)-F^{\prime}\left(x_{k}\right)\right] .
$$

Если в этом выражении принять $\beta=1$ и в формуле (5) положить $A_{k}=\Gamma_{k}$ и $V_{k}=Q_{k}^{-1}$, где $Q_{k}=\Gamma_{k} F^{\prime}\left(x_{k}-\frac{1}{2} \Gamma_{k} F\left(x_{k}\right)\right)$, то из (5) получается метод, рассмотренный Т. И. Коган $\left[{ }^{7}\right]$ 


$$
x_{k+1}=x_{k}-\left[F^{\prime}\left(x_{k}-\frac{1}{2} \Gamma_{k} F\left(x_{k}\right)\right)\right]^{-1} F\left(x_{k}\right), \quad k=0,1, \ldots .
$$

Если в методе (12) заменить $\Gamma_{k}$ на $\left[F^{\prime}\left(\theta_{k-1}\right)\right]^{-1}$ и $\left[F^{\prime}\left(x_{k}-\right.\right.$ $\left.\left.-\frac{1}{2} \Gamma_{k} F\left(x_{k}\right)\right)\right]^{-1}$ на $\left[F^{\prime}\left(\theta_{k}\right)\right]^{-1}$, где

$$
\theta_{k}=\left\{\begin{array}{l}
x_{0}, \quad \text { при } \quad k=0 \\
x_{k}-\frac{1}{2}\left[F^{\prime}\left(\theta_{k-1}\right]^{-1} F\left(x_{k}\right), \quad \text { при } k \geqslant 1,\right.
\end{array}\right.
$$

то (12) превращается в метод

$$
x_{k+1}=x_{k}-\left[F^{\prime}\left(\theta_{k}\right)\right]^{-1} F\left(x_{k}\right)
$$

с порядком $1+\sqrt{2}$. В самом деле,

$$
\begin{aligned}
& \left\|I-A_{k} F^{\prime}\left(x_{k}\right)\right\| \leqslant \|\left[F^{\prime}\left(\theta_{k-1}\right)\right]^{-1}\left(F^{\prime}\left(\theta_{k-1}\right)-F^{\prime}\left(x_{k}\right)\|\leqslant C K\| x_{k}-\theta_{k-1} \| \leqslant\right. \\
\leqslant & C K\left(\left\|x_{k}-x_{k-1}\right\|+\frac{1}{2}\left\|\left[F^{\prime}\left(\theta_{k-2}\right)\right]^{-1} F^{\prime}\left(\theta_{k-1}\right)\right\|\left\|\left[F^{\prime}\left(\theta_{k-1}\right)\right]^{-1} F\left(x_{k-1}\right)\right\|\right),
\end{aligned}
$$

т. e. $\gamma_{k}=0\left(\left\|x_{k}-x_{k-1}\right\|\right)$.

С учетом того, что $U_{k}=A_{k} F^{\prime}\left(x_{k}-\frac{1}{2} A_{k} F\left(x_{k}\right)\right)$ и $V_{k}=U_{k}^{-1}=$ $=\left[F^{\prime}\left(\theta_{k}\right)\right]^{-1} A_{k}^{-1}$, имеем $\left\|I-V_{k} U_{k}\right\|=0$, т. е. $\bar{\gamma}_{k}=0$. Далее, из (11) следует, что доминирующим является член $\frac{1}{2} \Lambda K_{\gamma_{k-1}}\left\|x_{k}-x_{k-1}\right\|^{2}$ порядка $O\left(\left\|x_{k-1}-x_{k-2}\right\|\left\|x_{k}-x_{k-1}\right\|^{2}\right)$, и поэтому скорость сходимости метода (13) равна $1+\sqrt{2}\left[{ }^{8}\right]$.

По-видимому, метод (13) впервые предложен М. Я. Бартишем [9 $]$, позже рассмотрен в $\left[{ }^{10-15}\right]$.

Применение метода (13) равносильно решению на каждом шаге итерации возмущенных линейных уравнений

$$
\begin{aligned}
\left(F^{\prime}\left(x_{k}\right)+E_{k}\right) t_{k} & =-F\left(x_{k}\right), \\
F^{\prime}\left(x_{k}+\frac{1}{2} t_{k}\right) \tau_{k} & =-F\left(x_{k}\right),
\end{aligned}
$$

где $x_{k+1}=x_{k}+\tau_{k} \quad$ и $E_{k}=\left[F^{\prime}\left(\theta_{k-1}\right)\right]^{-1}-F^{\prime}\left(x_{k}\right)$, а применение метода (12) эквивалентно решению (14), (15) при $E_{k}=0$. Если при реализации метода (13) использовать схему с вычислением обратного оператора, то на каждом шаге итерации требуется приблизительно такой же объем вычислений, как и при методе Ньютона.

На основе изложенного выше заключаем, что для сохранения порядка, присущего методу Ньютона или методу (13), следует вычислить обратные операторы или соответствующие линейные уравнения с точностью $O\left(\delta^{2^{k}}\right)$ или $O\left(\delta^{(1+\sqrt{2})^{k}}\right)$. При решении на каждом шаге итерации линейных уравнений с помощью некоторого итерационного метода для сохранения порядка метода (12) (метода (13)) достаточно решить уравнение (14) с точностью порядка $O\left(\left\|x_{k+1}-x_{k}\right\|\right)\left(O\left(\| x_{k}-x_{k-1}\right)\right)$, а уравнение (15) с точностью $O\left(\left\|x_{k+1}-x_{k}\right\|^{2}\right)\left(O\left(\left\|x_{k}-x_{k-1}\right\|\left\|x_{k+1}-x_{k}\right\|\right)\right)$ (см. также $\left.\left[{ }^{1}\right]\right)$.

В частности, для решения уравнений $(14),(15)$ можно использовать двупараметрический итерационный метод [ $\left.{ }^{16-18}\right]$ 


$$
x_{k+1}=x_{k}-\varepsilon_{k}\left(2 \alpha_{k}\left[F^{\prime}\left(x_{k}\right)\right]^{*}-\alpha_{k}^{2}\left[F^{\prime}\left(x_{k}\right)\right]^{*} F^{\prime}\left(x_{k}\right)\left[F^{\prime}\left(x_{k}\right)\right]^{*}\right) F\left(x_{k}\right) .
$$

Удобный способ определения параметров $h_{1}^{(k)}$ и $h_{2}^{(k)}\left(h_{1}^{(k)}=2 \varepsilon_{k} \alpha_{k}, h_{2}^{(k)}=\right.$ $\left.=\varepsilon_{k} \alpha_{k}^{2}\right)$ предложен в [18]. Там же показано, что метод (16) эффективнее метода Фридмана в смысле как количества итераций, так и машинного времени.

При решении систем нелинейных уравнений полезно в некоторых случаях (желательно иметь $\Gamma(x)$ в явном виде, распараллеливать вычисления и т. д.) использовать модификации метода (12), в которых $\Gamma_{k}$ или $\Gamma_{k-\frac{1}{2}}\left(\Gamma_{k-\frac{1}{2}}=\left[F^{\prime}\left(x_{k}-\frac{1}{2} \Gamma_{k} F\left(x_{k}\right)\right)\right]^{-1}\right)$ вычисляются точно на некоторых шагах или этапах итерации, а на промежуточных шагах или этапах применяется формула итеративного обращения матрицы. зом:

Например, метод (12) можно модифицировать следующим обра-

$$
\begin{gathered}
y_{k}=x_{k}-\frac{1}{2} \Gamma_{k} F\left(x_{k}\right), \\
x_{k+1}=x_{k}-\left[3 \Gamma_{k}-3 \Gamma_{k} F^{\prime}\left(y_{k}\right) \Gamma_{k}+\Gamma_{k}\left(F^{\prime}\left(y_{k}\right) \Gamma_{k}\right)^{2}\right] F\left(x_{k}\right),
\end{gathered}
$$

или

$$
\begin{aligned}
& y_{0}=x_{0}-\frac{1}{2} \Gamma_{0} F\left(x_{0}\right), \\
& x_{1}=x_{0}-\Gamma_{0-\frac{1}{2}} F\left(x_{0}\right), \\
& y_{k}=x_{k}-\frac{1}{2} \Gamma_{(k-1)-\frac{1}{2}} \sum_{i=0}^{q-1}\left(I-F^{\prime}\left(x_{k}\right) \Gamma_{(k-1)-\frac{1}{2}}\right)^{i} F\left(x_{k}\right), \quad q \geqslant 2, \\
& x_{k+1}=x_{k}-\Gamma_{k-\frac{1}{2}} F\left(x_{k}\right), \quad k \geqslant 1 .
\end{aligned}
$$

Для метода (13) получим аналогично

$$
\begin{aligned}
& x_{k+1}=x_{k}-A_{k+1} F\left(x_{k}\right), \quad A_{k+1}=\left[F^{\prime}\left(\theta_{k}\right)\right]^{-1}, \\
& \theta_{k+1}=x_{k+1}-\frac{1}{2} A_{k+1} F\left(x_{k+1}\right), \\
& x_{k+2}=x_{k+1}-A_{k+1} \sum_{i=0}^{p-1}\left(I-F^{\prime}\left(\theta_{k+1}\right) A_{k+1}\right)^{i} F\left(x_{k+1}\right), \\
& \theta_{k+2}=x_{k+2}-\frac{1}{2} A_{k+1} \sum_{i=0}^{q-1}\left(I-F^{\prime}\left(\theta_{k+1} A_{k+1}\right)^{i} F\left(x_{k+2}\right), \quad(p, q \geqslant 2) .\right.
\end{aligned}
$$

Расчеты по методу Ньютона и по методам (12), (17)-(19) были проведены на наборе тестовых задач, который содержит комплект тестзадач Аргонского Национального Центра, задачу ФрейденштейнаРота и задачу Бокса $\left[{ }^{19}\right]$. Счет велся на ЭВМ ЕС 1060 с удвоенной точностью до тех пор, пока не достигалась точность $\left\|x_{k+1}-x_{k}\right\| \leqslant 10^{-6}$ или $\left\|x_{k+1}-x_{k}\right\| \leqslant 10^{-9}$. Программы были составлены на языке ФОРТРАН. Результаты численных экспериментов с условием окончания расчетов $\left\|x_{k+1}-x_{k}\right\| \leqslant 10^{-9}$ приведены в таблице. 


\begin{tabular}{|c|c|c|c|c|c|c|}
\hline $\begin{array}{l}\text { Номер } \\
\text { задачи }\end{array}$ & $\begin{array}{c}\text { Метод } \\
\text { Ньюто- } \\
\text { на }\end{array}$ & $\begin{array}{c}\text { Метод } \\
\text { (12) }\end{array}$ & $\begin{array}{l}\text { Метод } \\
\text { (13) }\end{array}$ & $\begin{array}{c}\text { Метод } \\
\text { (17) }\end{array}$ & $\begin{array}{c}\text { Метод } \\
(18) \\
q=3\end{array}$ & $\begin{array}{c}\begin{array}{c}\text { Метод } \\
(19) \\
p=q=3\end{array} \\
\end{array}$ \\
\hline 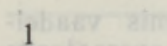 & 3 & 2 & 3 & 2 & 2 & 3 \\
\hline $\begin{array}{l}1 \\
2\end{array}$ & 32 & 21 & 27 & 21 & 22 & 42 \\
\hline 3 & 13 & 9 & 12 & 9 & 10 & 13 \\
\hline 4 & 15 & 28 & 14 & 24 & 37 & 16 \\
\hline 5 & 11 & 6 & - & 8 & - & - \\
\hline $6(N=6)$ & 13 & 9 & 15 & 9 & 9 & 110 \\
\hline $6(N=9)$ & 14 & 10 & 16 & 11 & 10 & - \\
\hline & 6 & 4 & 6 & 5 & 4 & - \\
\hline 8 & 91 & $K>300$ & 2 & - & 3 & - \\
\hline 9 & 4 & 3 & 4 & 3 & 3 & 5 \\
\hline $10(N=1)$ & 4 & 3 & 4 & 3 & 3 & 5 \\
\hline $10(N=10)$ & 4 & 3 & 4 & 3 & 3 & 5 \\
\hline & 8 & 7 & 12 & $K>300$ & $K>300$ & - \\
\hline 12 & 15 & 10 & 13 & 10 & 11 & 12 \\
\hline 13 & 5 & 4 & 5 & 4 & 4 & 7 \\
\hline 14 & 7 & 5 & 6 & 5 & 5 & 9 \\
\hline 15 & 43 & 25 & 10 & 60 & 49 & 17 \\
\hline 16 & 6 & 4 & 5 & 4 & 4 & 8 \\
\hline
\end{tabular}

$N$ - размерность задачи, прочерк - процесс не сходился, $K>300-$ число итераций превышало 300 .

Автор благодарит Вильве Кулла за проведение расчетов на ЭВМ.

\section{ЛИ Т Е РА Т Р А}

1. Ваарманн О. О некоторых итерационных методах с последовательной аппроксимацией обратных и псевдообратных операторов. Автореф. канд. дис. Таллин, 1970.

2. Вержбицкий В. М., Цалюк З. Б. Ж. вычисл. мат. и мат. физ., 12, № 1, 222-227 (1972).

3. Бартищ М. Я., Сеньо П. С. Вычисл. и прикл. математика, вып. 28, 85-93 (1976).

4. Щербина Ю. Н. Вестн. Львовского ун-та. Сер. мех.-мат., вып, 11, 66-70 (1976).

5. Бельтюков Б. А., Зыкова З. П. В кн.: II симпозиум по методам решения нелинейных уравнений и задач оптимизации. Хаапсалу, 4-7 июня 1981 г. Доклады и сообщения. Ч. І. Таллин, «Валгус», 1981, 12-17.

6. Райзман А. И. В кн.: II симпозиум по методам решения нелинейных уравнений и задач оптимизации. Хаапсалу, 4-7 июня 1981 г. Доклады и сообщения. Ч. I. Таллин, «Валгус», 1981, 101-103.

7. Коган Т. И. Сиб. мат. журн., 8, № 4, 958-960 (1967).

8. Schmidt, J. W. Z. angew. Math. und Mech., B. 43, № 3, 97-110 (1963).

9. Бартии М. Я. ДАН УССР. Сер. А, № 5, 387-391 (1968).

10. Laasonen, P. Ann. Acad. Sci. Fenn. Ser. A. I., 450 (1969).

11. King, R. F. Numer. Math., 18, 298-304 (1972).

12. Werner, W. Numer. Math., 32, 333-342 (1979).

13. Werner, $W$. Numer. Math., 38, 383-392 (1982).

14. Щербина Ю. Н. Исследование некоторых итерационных методов решения нелинейных операторных уравнений. Автореф. канд. дис. Львов, 1975.

15. Роман Л. Л. В кн.: III симпозиум «Методы решения нелинейных уравнений и задач оптимизащии». Доклады и сообщения. Таллин, «Валгус», 1984, 90-91.

16. Ваарманн О. Изв. АН ЭССР. Физ. Матем., 19, № 3, 265-274 (1970).

17. Ваарманн O. В кн.: II симпозиум по методам решения нелинейных уравнений и задач оптимизации. Хаапсалу, 4-7 июня 1981 г. Доклады и сообщения. Ч. І. Таллин, «Валгус», 1981, 18-26.

18. Mărușter, St. On the two-step gradient method for nonlinear equations. Seminarul de Informatică Și Analiză Numerică. Timişoara, Universitatea Din Timișoara, Nr. 20, 1985.

19. Moré, J. J., Garbow, B. S., Hillström, K. E. ACM. Trans. Math. Software, 7, 17-41 (1981).

Институт кибернетики

Академии наук Эстонской ССР
Поступила в редакцию $24 / \mathrm{XI} 1986$ 


\section{PUUTUVATE HUPERBOOLIDE TUUPI MEETODITEST}

Mittelineaarse operaatorvõrrandi $F(x)=0$ lahendamiseks Banachi ruumis vaadeldakse puutuvate hüperboolide meetodi (2) selliseid modifikatsioone, kus operaatorite $F^{\prime \prime}(x), Q(x), \Gamma(x)$ asemel kasutatakse nende aproksimatsioone. On tōestatud koonduvusteoreem seda tüüpi iteratsioonimeetodite kohta, mis on tuletatud mitmel erinevál viisil. On toodud tingimused, mille täidetuse korral vaadeldavad meetodid säilitavad neile omase koonduvuskiiruse järgu. Teoreetiliste tulemuste illustreerimiseks on lahendatud 16 näitülesannet.

\section{O. VAARMANN}

\section{ON METHODS OF TANGENT HYPERBOLS TYPE}

For solving the equation $F(x)=0$, where $F(x)$ is twice differentiable mapping from a Banach space into another, one considers inexact methods of tangent hyperbols (2) and such where the operators $F^{\prime \prime}(x), Q(x)$ and $\Gamma(x)$ are replaced by their approximations. A local convergence theorem is proved for a family of inexact methods of tangent hyperbols derived by variational means. This theorem, too, states conditions under which methods preserve their convergence rate. As an illustration of new variants of method (2), numerical results of solving 16 test problems are presented. 